\title{
Computational Modelling in Cancer: Methods and Applications
}

\author{
Konstantina Kourou ${ }^{a}$, Dimitris I. Fotiadis ${ }^{b, c}(\bowtie)$ \\ a Dept. of Biological Applications and Technology, University of loannina, loannina, Greece \\ http://www.uoi.gr/en/science_technology2.php \\ ${ }^{b}$ Unit of Medical Technology and Intelligent Information Systems, Dept. of Materials Science and Engineering, \\ University of loannina, loannina, Greece \\ http://medlab.cs.uoi.gr/index.asp \\ c IMBB - FORTH, Dept. of Biomedical Research, loannina, Greece \\ http://www.imbb.forth.gr/en/
}

\section{ARTICLE INFO: \\ RECEIVED: 31 Oct 2014 \\ REVISED: 15 Dec 2014 \\ ACCEPTED: 23 Dec 2014 \\ ONLINE: 03 Jan 2015}

\section{KEYWORDS:}

Cancer informatics Computational Modelling

Petri Nets

Cellular Automata

Agent-based Systems

Differential Equations

Angiogenesis

\begin{abstract}
A BSTRACT
Computational modelling of diseases is an emerging field, proven valuable for the diagnosis, prognosis and treatment of the disease. Cancer is one of the diseases where computational modelling provides enormous advancements, allowing the medical professionals to perform in silico experiments and gain insights prior to any in vivo procedure. In this paper, we review the most recent computational models that have been proposed for cancer. Well known databases used for computational modelling experiments, as well as, the various markup language representations are discussed. In addition, recent state of the art research studies related to tumour growth and angiogenesis modelling are presented.
\end{abstract}

\section{Introduction}

Cancer, a broad term for a class of diseases, is characterised by abnormal cells growth that invades healthy cells in the body. Disease characteristics are well described; ${ }^{1,2}$ there are certain conditions that have to be fulfilled in order for cancer to grow and spread, and not only aberrant cellular growth.

Rapid advances in cancer research in the last decade revealed that cancer is a disease which includes dynamic genomic changes. A number of molecular, cellular and biochemical characteristics have been suggested as the acquired capabilities which are shared by almost all types of human cancers. A set of rules have been studied to provide the clues that control the transformation of normal cells into malignant tumours. ${ }^{2}$ In addition, a variety of published studies specify that tumour growth in humans is a multistage process which reveals the genetic mutations that govern the alteration of normal human cells. ${ }^{3-5}$ The list with the six hallmarks of cancer ${ }^{1}$ provides extra knowledge to better understand the biology of cancer. These fundamental traits, regarding the 
alterations in cell's physiology, are referred to: (i) the sustainment of proliferation signalling, (ii) the evasion of growth suppressors, (iii) the activation of invasion and metastasis, (iv) the ability of replicative immortality, (v) the induction of angiogenesis and (vi) the resistance to cell death.

Cancer initiation depends on a series of genetic mutations which affect intrinsic cellular processes. Moreover, tumours evolve based on a scheme of progression which includes a complex network of interactions among the cancer cells and between cancerous cells and their micro-environment., Cancer complexity refers not only to the complex network of interacting signalling pathways, but also to the additional levels of interactions such as those at the supra-cellular levels. Hence, the increased cancer complexity is based on the large number of interacting molecules, the information exchange between pathways and the non-linear connections between the molecules. This multiparametric functioning of a system defines cancer as a systems biology disease. Consequently, there is a recent trend within the cancer research community to study cancer as a complex biological system and predict its behaviour. A number of distinct cell types are involved in most tumours, while their assemblance constitutes the tumour microenvironment which consists of heterotypic signalling interactions. Concerning the vast amount of quantitative data which are generated from experimentalists and high-throughput experimental techniques, a systems approach is needed which permits their integration and interpretation. Computational modelling, ${ }^{8,9}$ refers to a set of computer-based tools that allow users to create and/or visualize a model of a given system. Computational modelling methods can be useful for a biologist or a physician if a number of conditions could be addressed. ${ }^{10}$ These requirements refer to the fact that: (i) predictions can be mapped to experimental measurements, (ii) experiments can be performed in silico, aiming to reduce time and cost, (iii) new insights of a system's processes can be gained and (iv) complex systems can be better understood in terms of their interacting components. Due to the complexity of biological systems such as cancer, it is evident that various modelling approaches can efficiently address issues related to the accurate prediction of a system's behaviour.

The development of in silico cancer models will allow researchers to simulate experimental results and predict new clinical therapies, as well as to make new medical hypotheses for further experi- mental investigation. ${ }^{11,12}$ Due to the fact that cancer growth and invasion do not fit a single biological scale, it is evident that the use of computational techniques for multiscale cancer modelling paved the way to their applications in clinical practices. ${ }^{13-15}$ Figure 1 depicts how the computational cancer modelling field has evolved over time. In order to retrieve sufficient results, advanced Pubmed ${ }^{16}$ search queries were performed which allowed to reveal the number of computational studies per year, concerning the computational development of cancer models.

According to Wooley and Lin, ${ }^{9}$ computational modelling techniques are applied to specific biological phenomena and these models are used to pursue a number of intentions. Biological models can be useful in: (i) generating accurate quantitative predictions, (ii) predicting variables of a system which are not accessible via experimental measurements as well as identifying key factors, (iii) interpreting data within a coherent framework and (iv) screening quantitative or descriptive hypotheses.

In this work, we discuss the various computational modelling approaches which have been proposed for modelling complex systems. More specifically, we present these modelling approaches which are applied in cancer while explaining also the different scales of cancer modelling. The computational aspects of the modelling approaches for tumour growth and angiogenesis are also described.

\section{Computational Modelling of Cancer}

Tumours can be characterized as complex systems. Due to their intrinsic complexity, computational models must be developed at different scales aiming to analyse their growth and invasion. When cancer models are developed across multiple biological scales, the increasing number of the model parameters as well as the relationships among their interacting components should be considered. Once a multiscale cancer model is designed, multiple hierarchies in space and time have to be integrated, namely: (i) atomic scale, (ii) molecular scale, (iii) microscopic and (iv) macroscopic scale. ${ }^{11}$

At the atomic level, modelling techniques are used to study the structure and dynamic properties of proteins, peptides and lipids. At this scale, molecular dynamics simulations are used widely as a modelling method for molecules and atoms which interact over time. ${ }^{17,18}$ Models which are developed at the molecular scale usually study cell 


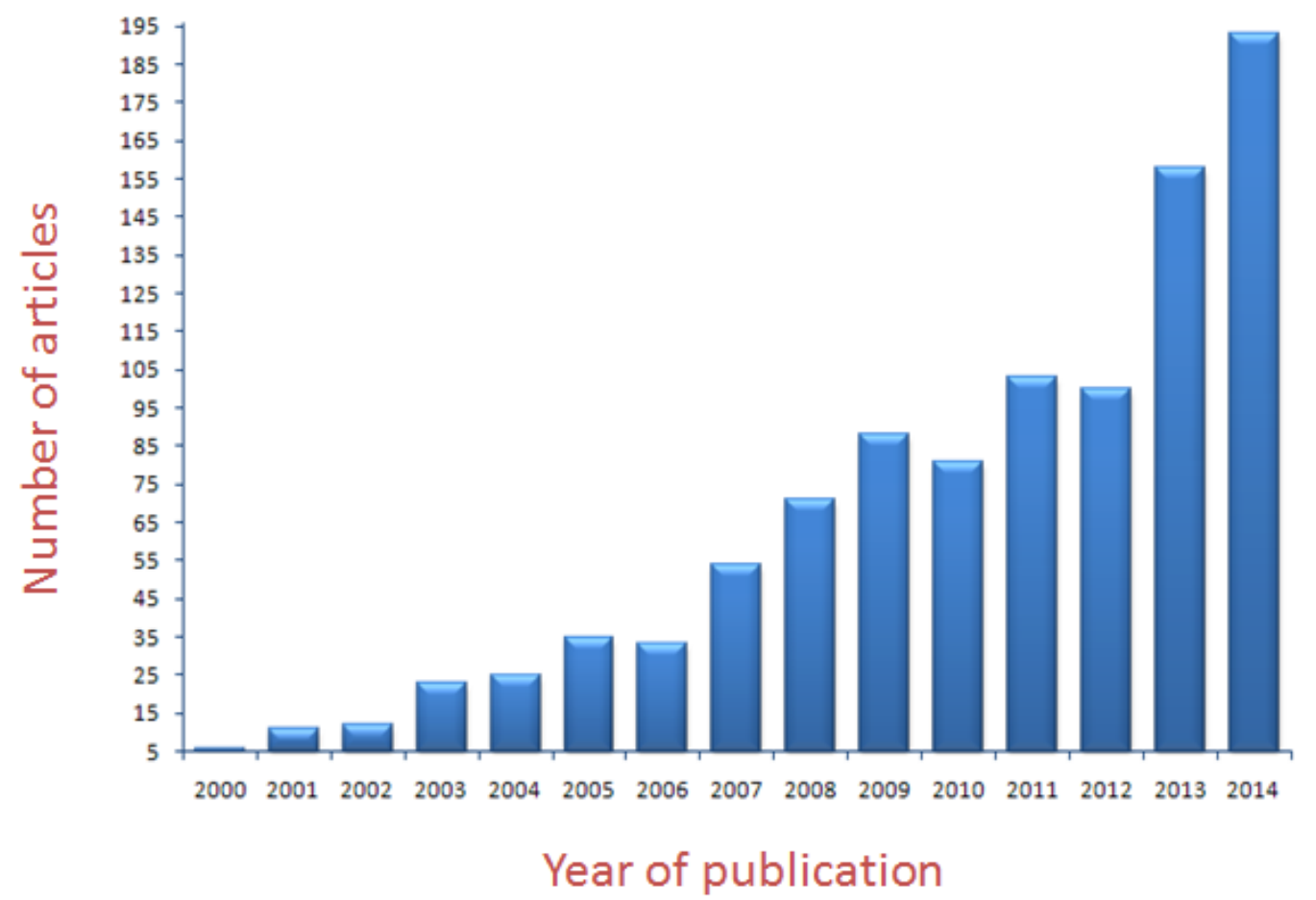

Figure 1: Results by year, regarding the number of published computational cancer modelling studies. Search queries in the PubMed biomedical database were performed for extracting the results (last visited 15 Dec 2014).

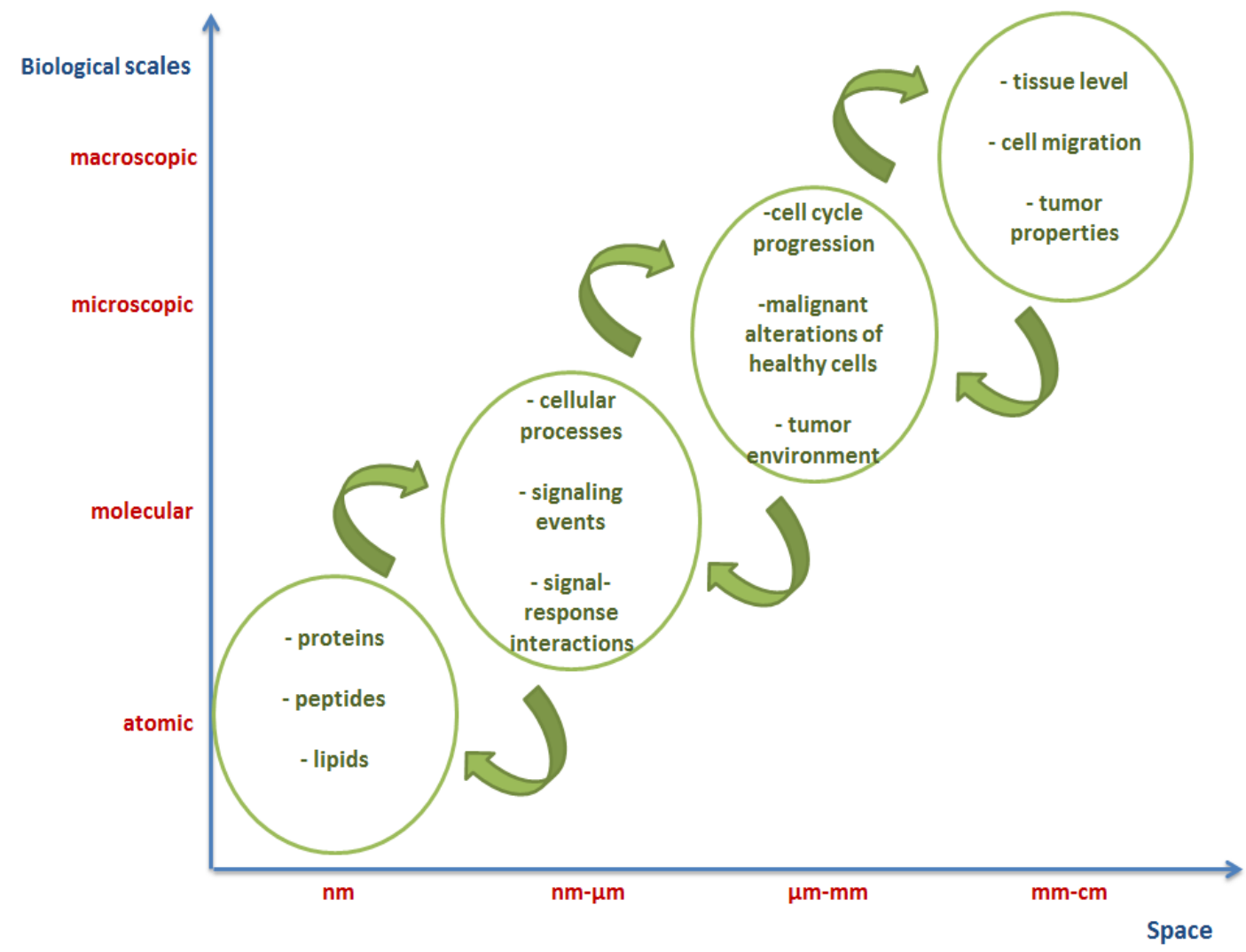

Figure 2: Illustration of the biological scales for computational cancer models including atomic, molecular, microscopic and macroscopic. Each scale represents different spatial range. Interconnection between the scales is required for the development of multiscale computational cancer models. 
signalling processes and refer to the properties of a set of proteins and not to individual molecules. ${ }^{18}$ In addition, models at the microscopic scale focus on the molecular and sub-cellular phenomena, like the cell cycle progression, which occur within a cell. It is also referred as the cellular scale that efficiently describes alterations in cell-cell interactions as well as the heterogeneous tumour environment. ${ }^{17,18}$ Lastly, the macroscopic scale refers to these biological processes that take place at the tissue level, such as cell migration. Computational models constructed in this level focus on the dynamic properties of the tumour's behaviour, including its shape and morphology under different environmental states. ${ }^{18}$ Figure 2 depicts the biological scales which are considered for the development of computational cancer models along with the investigations in each scale and their specific focus.

Computational models that study cancer behaviour often fall into two main categories, discrete and continuum. ${ }^{7,10,11}$ Discrete modelling represents each cell individually in space and time, based on a particular set of biophysical and biochemical procedures. In this category, processes like carcinogenesis, genetic instability and cell-cell interaction are usually studied. The second category is used for the modelling of large-scale systems. Continuum models study tumours as a collection of tissues. The integration of these two categories resulted in a new technique, namely hybrid modelling, ${ }^{19}$ which combines the advantages of both the discrete and continuum descriptions for modelling cancer at the molecular, cellular and tumour scales. ${ }^{10,20}$ One of the major advantages of hybrid models is that the participating entities of a system are better characterized and described due to the fact that they can progress from high-level descriptions to low-level details.

As mentioned above, computational tools have been widely used for representing complex biological systems. Although each model has been developed individually for addressing specific molecular issues, no attention has been paid on their integration. Based on that knowledge, a basic formalism, the Systems Biology Markup Language (SBML), has been developed for describing the biochemical processes and the networks which form within cells. ${ }^{21-23}$ The SBML markup language makes use of the eXtensive Markup Language (XML) for representing the components, reactions and parameters in a cell. ${ }^{24}$ The main motivations for the SBML creation were: (i) the use of a common file format in order to support different modelling tools, (ii) the ability to repeat experiments based on published models regardless of the modelling software used, and (iii) the promotion of developed models to the public in accordance to the current modelling tools.

During the International Union of Physiological Sciences (IUPS) of the Human Physiome Project, ${ }^{25}$ another XML-based language, CellML, ${ }^{26,27}$ was proposed for modelling the networks of interconnecting entities which are formed. CellML is based on mathematical equations to model electrophysiological systems as well as chemical reactions and networks. The purpose of CellML is to cover biological phenomena such as cell functions. Similar to CellML, insilicoML (ISML), is another markup language which provides users with a modular description of the constructed models. ${ }^{28} \mathrm{~A}$ variety of mathematical models, defined with ordinary differential equations (ODEs) and agent-based modelling approaches, are supported by the ISML language. A complement to CellML was the creation of the FieldML markup language which models physiological structures in terms of geometric fields. ${ }^{29}$ A number of SBML tools have been proposed which allow researchers to study systems biology computationally. ${ }^{30-34}$

In addition, specific online databases have been created for the storage of published computational models as well as of their parameters. Table 1 depicts the developed formalisms for representing biological systems along with their respective descriptions.

Table 1. Markup languages created for modelling and representing biological systems.

\begin{tabular}{|c|c|}
\hline $\begin{array}{l}\text { Markup } \\
\text { Language }\end{array}$ & Description \\
\hline $\mathrm{SBML}^{22}$ & $\begin{array}{l}\text { A domain-specific markup language } \\
\text { that describes biological processes } \\
\text { at the molecular level. }\end{array}$ \\
\hline CellML ${ }^{26,27}$ & $\begin{array}{l}\text { A modeling markup language for } \\
\text { modeling biological phenomena in } \\
\text { terms of mathematical equations. }\end{array}$ \\
\hline insilicoML ${ }^{28}$ & $\begin{array}{l}\text { A markup language that provides } \\
\text { modular description of models. }\end{array}$ \\
\hline FieldML ${ }^{29}$ & $\begin{array}{l}\text { A compliment to CellML markup } \\
\text { language for modeling physiological } \\
\text { structures. }\end{array}$ \\
\hline TumorML ${ }^{36}$ & $\begin{array}{l}\text { In silico cancer modeling markup } \\
\text { language. }\end{array}$ \\
\hline
\end{tabular}


According to Johnson et al., the development of a cancer-specific XML markup language will allow connecting model components for predictions by in silico oncology. ${ }^{35}$ Each of the proposed markup languages models different aspects in systems biology where researchers deal with more complex and multiscale behaviours. However, the general usage of these languages still remains an obstacle for cancer modelling. Thus, TumorML was developed as a new markup language for the computational modelling of cancer. ${ }^{36}$ The key functions of TumorML include: (i) the curation of cancer models, (ii) the computational interface within cancer models, and (iii) the interconnection between cancer models.

In the following sections we briefly discuss different modelling techniques used in systems biology to computationally analyse complex biological systems and their constituents.

\section{Petri Nets}

Petri Nets (PNs) is a modelling technique used to represent processes within a biological system. ${ }^{37-}$ ${ }^{39}$ A PN indicates a clarification of a graph model which consists of active and passive nodes that are distinct, as well as of movable objects which define the dynamic properties of a given system. The passive part includes a set of nodes called "places," while the active one refers to nodes called "transitions." The movable objects have been characterized as "tokens" which are transferred from one "place" to another based on specific "firing rules." These transitions depict the reactions which have occurred. They can be characterized as "fired" when enough sources of molecules exist while they produce products of the appropriate weights. One of the limitations of PN is the fact that they cannot be considered as a tool for qualitative simulations within exact time scales. However, the use of advanced PNs can "mask" other modelling techniques, such as systems of Ordinary Differential Equations. Their use has been expanded to cope with more complex issues resulting from other modelling approaches.

A variety of biological processes have been modelled by means of PNs. Among the examples are the modelling of apoptosis and the p53 transcription activity. The PN approach has been widely used for modelling many aspects in systems biology as well as for describing biological networks. ${ }^{40-43}$

\section{Cellular Automata}

Cellular Automata (CA) has been used to model individual molecules, as well as the rules that control their interactions. Cellular Automata refer to computer simulation tools used to model biological processes where time and space are discrete entities. With the invention of CA an infinite lattice of points (cells) were considered to have a finite number of states. ${ }^{44}$ In systems biology, lattice refers to two or three dimensional levels in space and the lattice-free systems correspond to real, physical space. Various studies of CA were conducted and focus on systems biology issues. ${ }^{44-47}$

Moreover, a cellular automaton model of tumour growth is proposed in which each cell corresponds to an environmental network. ${ }^{48,49}$ This network takes as input environmental parameters while the cellular behaviour is considered as the output. The authors also examined the impact of oxygen concentration on the growth and dynamic changes of the tumour. Their results indicate a correlation between the oxygen concentrations and the tumour's growth dynamics.

\section{Agent-based Systems}

Agent-based ( $A B)$ systems consider genes, proteins or cells as autonomous "agents" that can interact with each other based on a set of rules, across time and space. In comparison to CA modelling techniques, $A B$ systems do not require synchronized time steps and consider the space in a lattice free grid. ${ }^{7}$ In addition, while CA represent cancer cells as individual entities, $A B$ models include different cell types, genetic elements and environmental factors which interact to each other and share divergent functionalities. The $A B$ modelling technique has been proven a powerful tool for representing discrete biological operations. It has been used to study multicellular procedures such as tissue electrical conduction, cell trafficking, tissue mechanics, arterial modelling and inflammation. ${ }^{50-55}$

Computational cancer models that have been introduced across molecular and microscopic scales have widely used $A B$ approaches. Athale, Mansury and Deisboeck developed a framework within a multiscale agent-based environment that allowed them to analyse the complex cellular behaviour of tumour growth, as well as to study the molecular profiles of the cells which change over time and space. ${ }^{56}$ Their results indicate that the developed model simulates tumour growth within a multiscale environment, which allows research- 
ers to further study molecular signatures, as well as multicellular patterns.

\section{Ordinary Differential Equations}

When the biological processes are presented as systems of chemical reactions, mathematical modelling techniques that are observed in chemistry are needed for their analysis. Studies of Ordinary Differential Equations (ODEs) form a wellknown computational approach in systems biology. ${ }^{7,10}$ ODEs have been used extensively to model a set of processes, including metabolic pathways, ${ }^{57}$ mitosis in yeast ${ }^{58,59}$ and genetic regulatory pathways. ${ }^{60}$ ODE refers to the equation that states the relationship between a function and its derivatives, while it defines how a variable changes over time. ${ }^{61-64}$ There are cases in chemistry modelling where kinetics is described according to logarithmic functions. Thus, for this specific class of modelling, power law estimations of ODE systems have been constructed. One of the basic drawbacks of ODE-based models is the fact that only one independent variable can be considered in a system.

Many ODE models have been developed regarding the changes of some chemical species across the independent variable time. On the other hand, Partial Differential Equations (PDEs) are used to model more explicitly the distribution of a system's variables. For example, Bresch et al. constructed a multiscale model using PDEs to analyse the evolution of cancer cells. ${ }^{65}$ The model was applied aiming to explore new therapeutic advantages while it can be further used for tumour growth simulations.

In the following two sections we discuss how the advances of computational modelling in systems biology allowed researchers to better understand the complex mechanisms of cancer progression: tumour growth and angiogenesis.

\section{Modelling Tumour Growth}

Disruption of the cell cycle may lead to uncontrolled proliferation of cells. The lack of limitations on growth allows the tumour to appear. Cancer growth can be divided into two stages: avascular and vascular stages. The difference between these two stages lies in the way the cells receive their nutrient and oxygen. In order to proliferate, cells can receive these components either from existing vascular vessels or from new blood vessels which are created near the tumours (angiogenesis). In addition, it is known that the cell cycle is controlled by the tumour growth microenvironment, which includes its shape, morphology and invasion. Although, the amount of experimental molecular data increased the last decade, little is known regarding the tumour growth, metastasis and treatment. As a result, modelling in microscopic and macroscopic scale will allow researchers to gain insights into the whole tumour behaviour and reveal key parameters which are involved in cancerous conditions.

A significant number of computational approaches to the modelling of tumour progression and invasion have been proposed. Most of these techniques model tumour's behaviour based on known characteristics, while they identify the factors that drive tumour progression towards invasion.

Hartung et al. assessed the significance of mathematical modelling which describes tumour's metastatic spreading, when no clinical outcome is available. ${ }^{66}$ The model consists of a transport estimation that describes metastatic growth in terms of a boundary condition for metastatic emission. The resulting predictive model, with the use of different computational approaches, was compared to specific experimental results. In addition, key biological factors were incorporated into the model in order to describe the tumour growth and metastasis. After the model's validation through residual analysis and a bootstrap study, significant findings were unveiled. The work defines tumour metastatic stages during the early diagnosis of cancer.

Furthermore, a number of studies addressed the overexpression of the Epidermal Growth Factor Receptor (EGFR), involved in malignant brain tumours. Deisboeck with colleagues constructed a multiscale $A B M$-based model aiming to find out possible connections between EGFR dynamics, malignant cell proliferation and cell migration. ${ }^{67} \mathrm{~A}$ system of ODEs was used to simulate the intracellular molecular interactions. It was also confirmed that a possible increase of the EGFR mass on the cell surface corresponds to a rise of tumour's expansion. According to Athale and Deisboeck, the integration of proteomics data to transcriptional analysis may reveal the aggressive tumour behaviour from proliferation to metastasis, as well as predict tumour dynamics. ${ }^{68}$ An alternative molecular model was also developed on the basis of PDEs, which led to the same findings, while suggesting the monitoring of protein-gene interactions in cancer cell phenotypes.

Masoudi-Nejad and co-authors presented multiscale approaches for cancer systems biology and 
modelling and discussed a variety of studies for computational modelling of tumour growth. ${ }^{7}$ The authors refer to specific works that: (i) model tumour growth factors by integrating cellular and tissue levels, ${ }^{69}$ (ii) make use of cellular Potts Model (CPM), a class of ABMs, that simulates molecular and biophysical connections between cells, ${ }^{70}$ and (iii) facilitate the simulation of cell and tissue shape changes by means of a lattice-free CA biophysical model. $^{71}$

Moreover, hybrid models were used extensively to study the tumour cell biology and further allow the integration of discrete and continuum variables regarding tumour growth. ${ }^{72-78}$

\section{Modelling Angiogenesis}

Concerning the tumour growth and metastasis, the process of angiogenesis arises when new blood capillaries are created next to the existing vessels. A number of growth factor complexes are involved in angiogenesis regulation, such as the vascular endothelial growth factor (VEGF). ${ }^{7}$

Based on ODEs simulations, a model has been developed that integrates vessel regression and vascular tumour growth. ${ }^{79}$ The model incorporates as key angiogenic factor the VEGF expression in tumours, while it also considers mature and immature vessels, based on their destabilization and regression, respectively.

Moreover, concerning the cellular response of hypoxia and the hypoxic cells that secret angiogenic factors, it was suggested that high levels of the hypoxia-inducible transcription factor (HIF1) is associated with higher grade gliomas. ${ }^{80,81}$ Qutub and Popel developed a model of the hypoxic response pathway based on ODEs. ${ }^{82}$ The model mirrors the molecular kinetics of 17 components and is validated using independent experimental datasets. Based on their findings, the authors suggest that iron supplementation combined with increased ascorbate could be a therapeutic technique in the case of HIF1 angiogenesis inhibition of hypoxic tumours. Regarding the model's limitations, the authors concluded that kinetic reaction rates and effects of acidic tumour microenvironment remained unknown.

Finley and Popel conducted an experimentbased study aiming to model VEGF kinetics and transport. ${ }^{83}$ It was suggested that anti-VEGF treatment occurring in tumour microenvironment could increase or decrease tumour VEGF. These findings reveal the significance of personalized medicine as the rate of VEGF could act as a possible biomarker.

\section{Discussion}

In this review, computational modelling techniques for cancer are presented along with their applications to specific biological processes, such as tumour growth and angiogenesis. The ability to model cancer initiation and invasion by means of a computational tool pave the way to improve cancer diagnosis, prognosis and treatment. In addition, computational cancer modelling allows researchers to investigate oncogenesis across multiple biological states.

In the last decade, mathematical and computational modelling approaches motivated experiments to generate predictions for clinical practices with the integration of quantitative experimental measurements. Based on that, cancer can be modelled as a system of biological processes and interacting parties and may reveal new insights into the systems biology field.

When computational models are developed without real experimental results and without parameterization and/or validation, no robust predictions of a system's behaviour can be made. Moreover, limitations regarding the model's applicability and validation should be addressed before the implementation of a constructed model. Researchers may face many challenges when trying to design their model and refine the selected computational approach. The choices of the model's parameters and the application of innovative multiscale methods are two of these challenges that should be in the focus of scientific research.

Issues regarding the computational demand and model reusability are also considered as serious challenges when developing predictive computational cancer models. Computational techniques that are used for this purpose should enable cancer models to be executed in an appropriate period of time as well as support its predictive power. Additionally, researchers should present their models in standardized formats in order to achieve model's reusability. Difficulties regarding the model's validation should be overcome to achieve higher predictive power. The creation of data repositories that allow the storage and exchange of developed computational models should also be considered aiming to efficiently provide researchers and clinicians with extra information and knowledge.

Individualized medicine refers to a medical model that makes use of patient's molecular profiling for discovering the right treatment strategy for the right person at the right time. Moreover, with the advent of the personalized healthcare, 
medical models may reveal the predisposition to complex diseases and the distribution of targeted disease prevention. With the construction of quantitative, predictive models for complex diseases, such as cancer, clinical and experimental data can be integrated resulting in improved personalized treatment.

Computational cancer modelling is among the most promising strategies as an effort to predict key parameters within cancer cells. With the completion of the Human Genome Project, recent advances in systems biology made possible for computational approaches to "attack" cancer across its genetic vulnerabilities. ${ }^{84}$

\section{Conclusions}

Computational cancer modelling is a promising research area for integrating systems biology knowledge as well as for revealing applications in clinical and basic research. Due to the complex system of cancer progression, there are many challenges and limitations that should be addressed by the researchers. The design and implementation of computational models should be based on the integration of quantitative clinical and experimental data. Several multiscale modelling techniques have been introduced in the field of computational modelling aiming to find out the best approaches that move the predictive models towards clinical practices. With the advent of computational tools for modelling complex systems, such as cancer, personalized medicine will be facilitated. Additionally, more benefits will be provided to the patients resulting in a more preventative healthcare system.

\section{References}

1 Hanahan D, Weinberg RA. The hallmarks of cancer. Cell. 2000 Jan 7;100(1):57-70. DOI: 10.1016/S00928674(00)81683-9.

2 Hanahan D, Weinberg RA. Hallmarks of cancer: The next generation. Cell. 2011 Mar 4;144(5):646-74. DOI: 10.1016/j.cell.2011.02.013.

3 Kinzler KW, Vogelstein B. Lessons from hereditary colorectal cancer. Cell. 1996 Oct 18;87(2):159-70. DOI: 10.1016/S0092-8674(00)81333-1.

4 Hahn WC, Counter CM, Lundberg AS, Beijersbergen $\mathrm{RL}$, Brooks MW, et al. Creation of human tumour cells with defined genetic elements. Nature. $1999 \mathrm{Jul}$ 29;400(6743):464-8. DOI: 10.1038/22780.

5 Bergers G, Hanahan D, Coussens L. Angiogenesis and apoptosis are cellular parameters of neoplastic progression in transgenic mouse models of tumorigenesis. Int J Dev Biol. 1998;42(7):995-1002. PMID: 9853830.
6 Hornberg JJ, Bruggeman FJ, Westerhoff HV, Lankelma J. Cancer: A systems biology disease. Biosystems. 2006 Feb-Mar;83(2-3):81-90. DOI: 10.1016/ j.biosystems.2005.05.014.

7 Masoudi-Nejad A, Bidkhori G, Hosseini Ashtiani S, Najafi A, Bozorgmehr JH, et al. Cancer systems biology and modeling: Microscopic scale and multiscale approaches. Semin Cancer Biol. 2015 Feb;30C:60-69. DOI: 10.1016/j.semcancer.2014.03.003.

8 Walpole J, Papin JA, Peirce SM. Multiscale computational models of complex biological systems. Annu Rev Biomed Eng. 2013;15:137-54. DOI: 10.1146/ annurev-bioeng-071811-150104.

9 Wooley JC, Lin HS. Computational modeling and simulation as enablers for biological discovery. In: Wooley JC, Lin HS, editors. Catalyzing Inquiry at the Interface of Computing and Biology. Washington (DC): National Academies Press (US); 2005. p. 117-202. Available from: http://www.ncbi.nlm.nih.gov/books/ NBK25466.

${ }^{10}$ Materi W, Wishart DS. Computational systems biology in cancer: Modeling methods and applications. Gene Regul Syst Bio. 2007 Sep 17; 1: 91-110. PMID: 19936081.

11 Deisboeck TS, Zhang L, Yoon J, Costa J. In silico cancer modeling: Is it ready for prime time? Nat Clin Pract Oncol. 2009 Jan;6(1):34-42. DOI: 10.1038/ncponc 1237.

12 Edelman LB, Eddy JA, Price ND. In silico models of cancer. Wiley Interdiscip Rev Syst Biol Med. 2010 JulAug;2(4):438-59. DOI: 10.1002/wsbm.75.

${ }^{13}$ Chakrabarti A, Verbridge S, Stroock AD, Fischbach $C$, Varner JD. Multiscale models of breast cancer progression. Ann Biomed Eng. 2012 Nov;40(11):2488-500. DOI: $10.1007 / \mathrm{s} 10439-012-0655-8$.

${ }^{14}$ Carney TJ, Morgan GP, Jones J, McDaniel AM, Weaver M, Weiner B, Haggstrom DA. Using computational modeling to assess the impact of clinical decision support on cancer screening improvement strategies within the community health centers. J Biomed Inform. 2014 Oct;51:200-9. DOI: 10.1016/j.jbi.2014.05.012.

15 Taylor CA, Draney MT, Ku JP, Parker D, Steele BN, Wang K, Zarins CK. Predictive medicine: computational techniques in therapeutic decision-making. Comput Aided Surg. 1999;4(5):231-47. DOI: 10.3109/10929089 909148176.

${ }^{16}$ Lu Z. PubMed and beyond: a survey of web tools for searching biomedical literature. Database (Oxford). 2011 Jan 18;2011:baq036. DOI: 10.1093/database/baq 036.

17 Wang Z, Butner JD, Kerketta R, Cristini V, Deisboeck TS. Simulating cancer growth with multiscale agentbased modeling. Semin Cancer Biol. 2015 Feb;30C:7078. DOI: 10.1016/j.semcancer.2014.04.001.

${ }^{18}$ Deisboeck TS, Wang Z, Macklin P, Cristini V. Multiscale cancer modeling. Annu Rev Biomed Eng. 2011 Aug 15;13:127-55. DOI: 10.1146/annurev-bioeng071910-124729. 
19 McAdams HH, Shapiro L. Circuit simulation of genetic networks. Science. 1995 Aug 4;269(5224):6506. DOI: $10.1126 /$ science. 7624793 .

${ }^{20}$ Wang Z, Deisboeck TS. Computational modeling of brain tumors: discrete, continuum or hybrid? Scientific Modeling and Simulation SMNS. 2008 Apr;15(1-3):38193. DOI: $10.1007 / \mathrm{s} 10820-008-9094-0$.

${ }^{21}$ Hucka M, Finney A, Sauro HM, Bolouri H, Doyle JC, et al. The systems biology markup language (SBML): a medium for representation and exchange of biochemical network models. Bioinformatics. 2003 Mar 1;19(4): 524-31. DOI: 10.1093/bioinformatics/btg015.

${ }^{22}$ Hucka M. Systems Biology Markup Language (SBML). In: Dubitzky W, Wolkenhauer O, Cho K-H, Yokota $\mathrm{H}$, editors. Encyclopedia of Systems Biology. New York: Springer; 2013. p. 2057-63.

${ }^{23}$ Finney A, Hucka M. Systems biology markup language: Level 2 and beyond. Biochem Soc Trans. 2003 Dec;31(Pt 6):1472-3. PMID: 14641091.

${ }^{24}$ Webb K, White T. UML as a cell and biochemistry modeling language. Biosystems. 2005 Jun;80(3):283302. DOI: 10.1016/j.biosystems.2004.12.003.

${ }^{25}$ Hunter $P$, Robbins P, Noble D. The IUPS human physiome project. European journal of physiology, 2002;445(1):1-9. DOI: 10.1007/s00424-002-0890-1.

${ }^{26}$ Lloyd CM, Halstead MD, Nielsen PF. CellML: its future, present and past. Prog Biophys Mol Biol. 2004 Jun-Jul;85(2-3):433-50. DOI: 10.1016/j.pbiomolbio.200 4.01.004.

${ }^{27}$ Cuellar AA, Lloyd CM, Nielsen PF, Bullivant DP, Nickerson DP, et al. An overview of CellML 1.1, a biological model description language. Simulation 2003 Dec;79(12):740-7. DOI: 10.1177/0037549703040939.

${ }^{28}$ Asai $\mathrm{Y}$, Suzuki $\mathrm{Y}$, Kido $\mathrm{Y}$, Oka $\mathrm{H}$, Heien E, et al. Specifications of insilicoML 1.0: a multilevel biophysical model description language. J Physiol Sci. 2008 Dec; 58 (7):447-58. DOI: 10.2170/physiolsci.RP013308.

${ }^{29}$ Britten RD, Christie GR, Little C, Miller AK, Bradley $C$, et al. FieldML, a proposed open standard for the Physiome project for mathematical model representation. Med Biol Eng Comput. 2013 Nov;51(11):1191-207. DOI: $10.1007 / \mathrm{s} 11517-013-1097-7$.

${ }^{30}$ Machné R, Finney A, Müller S, Lu J, Widder S, Flamm C. The SBML ODE Solver Library: a native API for symbolic and fast numerical analysis of reaction networks. Bioinformatics. 2006 Jun 1;22(11):1406-7. DOI: 10.1093/bioinformatics/btl086.

${ }^{31}$ Shapiro BE, Hucka M, Finney A, Doyle J. MathSBML: a package for manipulating SBML-based biological models. Bioinformatics. 2004 Nov 1;20(16):2829-31. DOI: $10.1093 /$ bioinformatics/bth271.

${ }^{32}$ Keating SM, Bornstein BJ, Finney A, Hucka $M$. SBMLToolbox: an SBML toolbox for MATLAB users. Bioinformatics. 2006 May 15;22(10):1275-7. DOI: 10.1093/ bioinformatics/btl111.

${ }^{33}$ Zi Z, Klipp E. SBML-PET: a Systems Biology Markup Language-based parameter estimation tool. Bioinformatics. 2006 Nov 1;22(21):2704-5. DOI: 10.1093/ bioinformatics/bt|443.
${ }^{34}$ Wellock C, Chickarmane V, Sauro HM. The SBWMATLAB interface. Bioinformatics. 2005 Mar;21(6):8234. DOI: 10.1093/bioinformatics/bti110.

${ }^{35}$ Johnson D, McKeever S, Stamatakos G, Dionysiou $\mathrm{D}$, Graf $\mathrm{N}$, et al. Dealing with diversity in computational cancer modeling. Cancer Inform. 2013 May 7;12:11524. DOI: $10.4137 /$ CIN.S11583.

36 Johnson D, Cooper J, McKeever S. TumorML: Concept and requirements of an in silico cancer modelling markup language. Conf Proc IEEE Eng Med Biol Soc. 2011;2011:441-4. DOI: 10.1109/IEMBS.2011.6090060.

37 Pinney JW, Westhead DR, McConkey GA. Petri Net representations in systems biology. Biochem Soc Trans. 2003 Dec;31(Pt 6):1513-5. PMID: 14641101.

38 Reddy VN, Mavrovouniotis ML, Liebman MN. Petri net representations in metabolic pathways. Proc Int Conf Intell Syst Mol Biol. 1993;1:328-36. PMID: 7584354.

39 Bonzanni N, Feenstra KA, Fokkink W, Heringa J. Petri Nets Are a Biologist's Best Friend. In: Fages F, Piazza C., editors, Formal Methods in Macro-Biology: Springer;2014. p. 102-16. DOI: 10.1007/978-3-31910398-3_8.

40 Genrich HJ, Lautenbach K. System modelling with high-level Petri nets. Theoretical computer science 1981;13(1):109-35. DOI: 10.1016/0304-3975(81)901134.

41 Voss K, Heiner M, Koch I. Steady state analysis of metabolic pathways using Petri nets. In Silico Biol. 2003;3(3):367-87. PMID: 14700469.

42 Sackmann A, Heiner M, Koch I. Application of Petri net based analysis techniques to signal transduction pathways. BMC Bioinformatics. 2006 Nov 2;7(1):482. DOI: 10.1186/1471-2105-7-482.

${ }^{43}$ Chaouiya C. Petri net modelling of biological networks. Brief Bioinform. 2007 Jul;8(4):210-19. DOI: 10.1093/bib/bbm029.

44 Ermentrout GB, Edelstein-Keshet L. Cellular automata approaches to biological modeling. J Theor Biol. 1993 Jan 7;160(1):97-133. DOI: 10.1006/jtbi.1993. 1007.

${ }^{45}$ Kier LB, Cheng CK, Testa B, Carrupt PA. A cellular automata model of diffusion in aqueous systems. J Pharm Sci. 1997 Jul;86(7):774-8. DOI: 10.1021/js970 0513.

46 Kier LB, Cheng C-K, Testa B, Carrupt P-A. A cellular automata model of enzyme kinetics. J Mol Graph. 1996 Aug;14(4):227-31, 226. PMID: 9076636.

47 Moreira J, Deutsch A. Cellular automaton models of tumor development: a critical review. Advs. Complex Syst. 2002;5(2-3):247-67. DOI: 10.1142/S02195259020 00572.

${ }^{48}$ Gerlee P, Anderson AR. An evolutionary hybrid cellular automaton model of solid tumour growth. J Theor Biol. 2007 Jun 21;246(4):583-603. DOI: 10.1016/ j.jtbi.2007.01.027.

${ }^{49}$ Gerlee P, Anderson AR. A hybrid cellular automaton model of clonal evolution in cancer: the emergence of the glycolytic phenotype. J Theor Biol. 
Feb 21,2008;250(4):705-22. DOI: 10.1016/j.jtbi.2007. 10.038 .

${ }^{50}$ Folcik VA, Broderick G, Mohan S, Block B, Ekbote C, et al. Using an agent-based model to analyze the dynamic communication network of the immune response. Theor Biol Med Model. 2011 Jan 19;8:1. DOI: 10.1186/1742-4682-8-1.

${ }^{51}$ Brown BN, Price IM, Toapanta FR, DeAlmeida DR, Wiley $C A$, et al. An agent-based model of inflammation and fibrosis following particulate exposure in the lung. Math Biosci. 2011 Jun;231(2):186-96. DOI: 10.1016/ j.mbs.2011.03.005.

52 Thorne BC, Hayenga HN, Humphrey JD, Peirce SM. Toward a multi-scale computational model of arterial adaptation in hypertension: verification of a multi-cell agent based model. Front Physiol. 2011 May 9;2:20. DOI: 10.3389/fphys.2011.00020.

${ }^{53}$ Das A, Lauffenburger D, Asada H, Kamm RD. A hybrid continuum-discrete modelling approach to predict and control angiogenesis: analysis of combinatorial growth factor and matrix effects on vessel-sprouting morphology. Philos Trans A Math Phys Eng Sci. 2010 Jun 28;368(1921):2937-60. DOI: 10.1098/rsta.2010.00 85.

${ }^{54}$ Artel A, Mehdizadeh H, Chiu Y-C, Brey EM, Cinar A. An agent-based model for the investigation of neovascularization within porous scaffolds. Tissue Eng Part A. 2011 Sep;17(17-18):2133-41. DOI: 10.1089/ten. TEA.2010.0571.

${ }^{55}$ Bentley K, Gerhardt H, Bates PA. Agent-based simulation of notch-mediated tip cell selection in angiogenic sprout initialisation. J Theor Biol. 2008 Jan 7;250(1):25-36. DOI: 10.1016/j.jtbi.2007.09.015.

${ }^{56}$ Athale C, Mansury Y, Deisboeck TS. Simulating the impact of a molecular 'decision-process' on cellular phenotype and multicellular patterns in brain tumors. J Theor Biol. 2005 Apr 21;233(4):469-81. DOI: 10.1016/ j.jtbi.2004.10.019.

${ }^{57}$ Ideker T, Thorsson V, Ranish JA, Christmas R, Buhler J, et al. Integrated genomic and proteomic analyses of a systematically perturbed metabolic network. Science. 2001 May 4;292(5518):929-34. DOI: 10.1126/ science.292.5518.929.

${ }^{58}$ Tyson JJ. Modeling the cell division cycle: $\mathrm{cdc} 2$ and cyclin interactions. Proc Natl Acad Sci U S A. 1991 Aug 15;88(16):7328-32. DOI: 10.1073/pnas.88.16.7328.

${ }_{59}$ Aguda BD. Modeling the cell division cycle. In: Friedman A, editor. Tutorials in Mathematical Biosciences III. Berlin: Springer;2006. p. 1-22.

${ }^{60}$ Elowitz MB, Leibler S. A synthetic oscillatory network of transcriptional regulators. Nature. 2000 Jan 20;403(6767):335-8. DOI: 10.1038/35002125.

${ }^{61}$ Kitano H. Systems biology: a brief overview. Science. 2002 Mar 1;295(5560):1662-4. DOI: 10.1126/ science.1069492.

${ }^{62}$ Kitano H. Computational systems biology. Nature. 2002 Nov 14;420(6912):206-10. DOI: 10.1038/nature 01254.
${ }^{63}$ Suresh Babu CV, Joo Song E, Yoo YS. Modeling and simulation in signal transduction pathways: a systems biology approach. Biochimie. 2006 Mar-Apr;88(34):277-83. DOI: 10.1016/j.biochi.2005.08.006.

${ }^{64}$ Kirschner MW. The meaning of systems biology. Cell. 2005 May 20;121(4):503-4. DOI: 10.1016/j.cell. 2005.05.005.

${ }^{65}$ Bresch D, Colin T, Grenier E, Ribba B, Saut O. Computational modeling of solid tumor growth: the avascular stage. SIAM J. Sci. Comput. 2010 Aug 4;32(4): 2321-44. DOI: 10.1137/070708895.

${ }^{66}$ Hartung N, Mollard S, Barbolosi D, Benabdallah A, Chapuisat $\mathrm{G}$, et al. Mathematical modeling of tumor growth and metastatic spreading: validation in tumorbearing mice. Cancer Res. 2014 Nov 15;74(22):6397407. DOI: 10.1158/0008-5472.CAN-14-0721.

${ }^{67}$ Deisboeck TS, Zhang L, Martin S. Advancing cancer systems biology: introducing the Center for the Development of a Virtual Tumor, CViT. Cancer Inform. 2007 Mar 30;5:1-8. PMID: 19390664.

${ }^{68}$ Athale CA, Deisboeck TS. The effects of EGFreceptor density on multiscale tumor growth patterns. J Theor Biol. 2006 Feb 21;238(4):771-9. DOI: 10.1016/ j.jtbi.2005.06.029.

${ }^{69}$ Ferreira S, Martins M, Vilela M. Reaction-diffusion model for the growth of avascular tumor. Phys Rev E. 2002 Jan 23;65(2 Pt 1):021907. DOI: 10.1103/PhysRev E.65.021907.

${ }^{70}$ Szabó A, Merks RM. Cellular potts modeling of tumor growth, tumor invasion, and tumor evolution. Front Oncol. 2013 Apr 16;3:87. DOI: 10.3389/fonc. 2013.00087.

${ }^{71}$ Galle J, Loeffler M, Drasdo D. Modeling the effect of deregulated proliferation and apoptosis on the growth dynamics of epithelial cell populations in vitro. Biophys J. 2005 Jan;88(1):62-75. DOI: 10.1529/biophysj. 104.041459 .

72 Mallet DG, De Pillis LG. A cellular automata model of tumor-immune system interactions. J Theor Biol. 2006 Apr 7;239(3):334-50. DOI: 10.1016/j.jtbi.2005.08. 002.

${ }^{73}$ Zheng $X$, Wise S, Cristini V. Nonlinear simulation of tumor necrosis, neo-vascularization and tissue invasion via an adaptive finite-element/level-set method. Bull Math Biol. 2005 Mar;67(2):211-59. DOI: 10.1016/j. bulm.2004.08.001.

${ }^{74}$ Frieboes HB, Lowengrub JS, Wise $\mathrm{S}$, Zheng $\mathrm{X}$, Macklin $\mathrm{P}$, et al. Computer simulation of glioma growth and morphology. Neuroimage. 2007;37 Suppl 1:S59-70. DOI: 10.1016/j.neuroimage.2007.03.008.

${ }^{75}$ Wise SM, Lowengrub JS, Frieboes HB, Cristini V. Three-dimensional multispecies nonlinear tumor growth-I: model and numerical method. J Theor Biol. 2008 Aug 7;253(3):524-43. DOI: 10.1016/j.jtbi.2008.03. 027.

${ }^{76}$ Rejniak KA, Anderson AR. Hybrid models of tumor growth. Wiley Interdiscip Rev Syst Biol Med. 2011 JanFeb;3(1):115-25. DOI: 10.1002/wsbm.102. 
77 Kim Y, Stolarska MA, Othmer HG. A hybrid model for tumor spheroid growth in vitro I: theoretical development and early results. Math Mod Meth Appl S. 2007;17(supp01):1773 - 98. DOI: 10.1142/S0218202507 002479.

${ }^{78}$ Macklin P, McDougall S, Anderson AR, Chaplain $M A$, Cristini $V$, et al. Multiscale modelling and nonlinear simulation of vascular tumour growth. J Math Biol. 2009 Apr;58(4-5):765-98. DOI: 10.1007/s00285-008-02 16-9.

${ }^{79}$ Arakelyan L, Vainstein V, Agur Z. A computer algorithm describing the process of vessel formation and maturation, and its use for predicting the effects of anti-angiogenic and anti-maturation therapy on vascular tumor growth. Angiogenesis. 2002;5(3):20314. DOI: 10.1023/A:1023841921971.

80 Semenza GL. Expression of hypoxia-inducible factor 1: mechanisms and consequences. Biochem Pharmacol. 2000 Jan 1;59(1):47-53. DOI: 10.1016/S0006-2952(99)0 0292-0.
81 Zagzag D, Zhong H, Scalzitti JM, Laughner E, Simons JW, Semenza GL. Expression of hypoxia-inducible factor $1 \alpha$ in brain tumors : association with angiogenesis, invasion, and progression. Cancer. 2000 Jun 1;88(11): 2606-18. DOI: 10.1002/1097-0142(20000601)88:11 <2606::AID-CNCR25>3.0.CO;2-W.

${ }^{82}$ Qutub AA, Popel AS. A computational model of intracellular oxygen sensing by hypoxia-inducible factor HIF1 $\alpha$. J Cell Sci. 2006 Aug 15;119(Pt 16):3467-80. DOI: $10.1242 /$ jcs.03087.

${ }^{83}$ Finley SD, Popel AS. Effect of tumor microenvironment on tumor VEGF during anti-VEGF treatment: systems biology predictions. J Natl Cancer Inst. 2013 Jun 5;105(11):802-11. DOI: 10.1093/jnci/djt093.

${ }^{84}$ Collins FS, Barker AD. Mapping the cancer genome. Pinpointing the genes involved in cancer will help chart a new course across the complex landscape of human malignancies. Sci Am. 2007 Mar;296(3):50-7. PMID: 17348159. 